\title{
Conversion of Banana Synseed Influenced by the Bead Type and Seed Coat
}

\author{
Ahmed Hassanein ${ }^{1}$, Ibrahim A. Ibraheim ${ }^{2}$, Abel-Naser Galal ${ }^{1}$, Jehan Salem ${ }^{1}$ \\ ${ }^{1}$ Central Laboratory of Genetic Engineering, Faculty of Science, Sohag University, Sohag, Egypt; ${ }^{2}$ Genetic Engineering and Biotech- \\ nical Laboratory, Sadat City, Monofia University, Monofia, Egypt. \\ Email: \{hassaneinam, ahmosman2000\}@yahoo.com
}

Received April 26 ${ }^{\text {th }}, 2011$; revised June 10 ${ }^{\text {th }}, 2011$; accepted June $23^{\text {rd }}, 2011$.

\begin{abstract}
Banana shoot tips extracted from in vitro grown plant materials were encapsulated in different types of alginate beads. The results obtained after conservation for one month at $4^{\circ} \mathrm{C}$ indicated that single layered synseeds (where the encapsulated shoot tip was covered by one layer of the artificial endosperm) containing activated charcoal were the best construction in stimulating conversion of synseeds, where they expressed the highest; conversion frequency, number of shoots/synseed, length of shoot and fresh weight/shoot cluster. These parameters decreased when the shoot tips were encapsulated within two layers of endosperm, except the ex vitro conversion. Conversion of banana synseeds was influenced by explant orientation inside the alginate beads, the highest conversion frequency was obtained when the shoot tip was directed upward inside the synthetic endosperm. Synthesis of seed coats around the artificial endosperm improved the conversion frequency, number of shoots/synseed and shoot length.
\end{abstract}

Keywords: Artificial Seeds, Banana, Conservation, Synseed Conversion, Tissue Culture

\section{Introduction}

Edible and cultivated banana cultivars are highly sterile, triploid and producing seedless fruits through parthenocary. These features prevent the implementation of breeding strategies to improve banana cultivars. The materials used for conventional propagation include: corms, large and small suckers, sward suckers $[1,2]$, transfer weevils, fungal pathogens, nematodes and viruses [2,3]. Compared to suckers, the use of plantlets obtained via tissue culture techniques (TC plantlets) express many additional advantages [4], where TC plantlets are cheaper and easier to propagate and transport. The uniformity of growth of in vitro obtained plants makes it possible to control the flowering time and harvesting. Also, they express higher survival rate in the field, and give a significant increase in yield and fruit quality $[5,6]$, but sumaclonal variation should be avoided $[7,8]$.

In fact, micropropagated plantlet production needs high investment [9] because the final products require specific and expensive management until commercialization and final delivery $[10,11]$. Development of synthetic seed production technology is currently considered an effective and efficient alternative method of propagation in several commercial important agronomic and hor- ticultured crops $[12,13]$. Synthetic seeds can be also called artificial seeds or synseeds. Somatic embryogenesis is considered the most appropriate technique for rapid-large scale micropropagation and synseed technology [14-16], but somatic embryogenesis technique is not well established in many plant species. In such cases, the encapsulation of shoot tips and/or axillary buds provides an alternative plant material to produce synseeds $[13,17]$. Only few reports described successful encapsulation of non-embryogenic in vitro-derived vegetative organs such as maxillary buds or shoot tips $[13,18]$.

Synseed consists of somatic embryo or alternative plant materials encapsulated within a synthetic endosperm which containing nutrient reserves and other additives [15,19-21]. These structures could also be encapsulated further with a synseed coats to suit mechanical handling and planting [22]. These synseeds can be stored for a longer period of time even up to 6 months without losing viability, especially when stored at $4^{\circ} \mathrm{C}$. In addition, to prevent embryo desiccation and mechanical injury, a number of useful materials such as fungicides, pesticides, antibiotics and microorganisms (e.g., rhizobia) can be included into the encapsulation matrix. Incorporation of activated charcoal improves the conversion and the vigor of encapsulated somatic embryos. 
Conversion ability of synseeds would be very necessary when ex vitro sowing is used [23]. In some species, such as banana, cardamom, mulberry and raspberry, encapsulated microcuttings demonstrated high adventitious rooting capacity after sowing [14,24,25], while other species did not respond $[23,26]$. There are many factors affect the conversion of synseeds after cold storage period. While, some factors need further studies, some others have not yet been studied, such as orientation of explants inside the artificial endosperm. Therefore, the aim of this work was to study factors affecting conversion of synseeds after cold storage on agar-solidified medium.

\section{Material and Methods}

\subsection{Explant Preparation and Culture Initiation}

Field grown plants ( $1.5 \mathrm{~m}-2.5 \mathrm{~m}$ high) of Hindi cultivar were obtained from Sohag Governorate and used as primary explants. The upper part of their shoots were cut back to approximately $30 \mathrm{~cm}$; roots and dry leaf sheaths were removed and transferred to the laboratory. Successive leaf bases of corms were cut away and the shoot tips were excised by making four angled cuts into the subtending tissue. The explants $(60 \mathrm{~mm} \times 20 \mathrm{~mm}$ cylinder $)$ were surface sterilized in $5.25 \% \mathrm{NaOCl}$ solution $(80 \%$ $\mathrm{v} / \mathrm{v}$ commercial Clorox bleach) for $20 \mathrm{~min}$ followed by 5 min dip in $0.2 \%(\mathrm{w} / \mathrm{v})$ mercuric chloride $\left(\mathrm{HgCl}_{2}\right)$, and rinsed three times in sterile distilled water. After disinfection, the excessive tissues were removed to get shoot tip explants of approximately $7 \mathrm{~mm} \times 6 \mathrm{~mm}$, including a basal corm tissue. These shoot tips were cut one time vertically in such a way to keep the base of the explants intact, and cultured on solid multiplication medium (MS medium supplemented with $5 \mathrm{mg} / \mathrm{l} \mathrm{BAP}$ ). The vegetative apices were transferred to fresh medium three times at two weeks intervals to avoid phenolic accumulation surrounding the cultured explants. When shoot multiplication began to form and continued growth warranted, they were subdivided into single shoots and subcultured on fresh multiplication media.

In vitro shoot tips $(4 \mathrm{~mm}-5 \mathrm{~mm}$ height and $2 \mathrm{~mm}-3$ $\mathrm{mm}$ at base) of banana cultivars (Hindi) derived from the initial stage were monthly subcultured on the multiplication medium supplemented with $5 \mathrm{mg} / \mathrm{l}$ BAP and 200 $\mathrm{mg} / \mathrm{l}$ ascorbic acid (ASA), or $0.2 \%$ activated charcoal as antioxidants. Ascorbic acid was added to the medium after autoclaving. In all experiments, MS [27] medium supplemented with $3 \%$ sucrose was used. Media were solidified with $4 \mathrm{gm} / \mathrm{l}$ agar (Difco Bacto agar) at $\mathrm{pH} 5.8$ before autoclaving. Vitamins (mg/l) were: myo-inositol (100), vitamin B1-hydrochloride (4), nicotinic acid (4), pyridoxal hydrochloride (0.7), biotin (0.04) and folic acid (0.5). Media were autoclaved at $121^{\circ} \mathrm{C}$ for 20 minutes. In vitro cultured plant materials were incubated under light condition of a tissue culture room $\left(25^{\circ} \mathrm{C} \pm 2^{\circ} \mathrm{C}\right.$ with $16 \mathrm{~h}$ photoperiod at $100 \mu \mathrm{mol} \cdot \mathrm{m}^{-2} \mathrm{~s}^{-1}$ ).

\subsection{Synseed Formation}

\subsubsection{Single Layered Beads}

Shoot multiplication medium with or without $2 \mathrm{gm} / \mathrm{l} \mathrm{ac}-$ tivated charcoal was used as artificial endosperm component. Sodium alginate $(4 \%)$ and $\mathrm{CaCl}_{2}(75 \mathrm{mM})$ solution were used as gel matrix and complexing agent, respectively. Both the gel matrix and complexing agent were autoclaved at $120^{\circ} \mathrm{C}$ for $20 \mathrm{~min}$ and stored at room temperature. Single layered beads were obtained by dipping the shoot tips in glass jar or Petri dish containing the gel matrix. Then, alginate-covered explants were picked up individually and dropped into the complexing agent for 30 minutes. Transfer time from gel matrix to complexing agent must not exceed five minutes. After hardening, the calcium chloride solution was decanted and the constructed alginate beads were rinsed three times with autoclaved liquid multiplication medium for $15 \mathrm{~min}$, $5 \mathrm{~min} / \mathrm{each}$, to wash away calcium chloride residues. The obtained beads (synseeds) were $5 \mathrm{~mm}-6 \mathrm{~mm}$ (Figure 1) in diameter and could be easily handled under sterile conditions without dehydration of the explant.

\subsubsection{Double Layered Beads with Explant in Bead Center}

Single layered synseeds (with or without $2 \mathrm{gm} / \mathrm{l}$ activated charcoal) were completely immersed in sodium alginate medium (with or without $2 \mathrm{gm} / 1$ activated charcoal). They were picked up and dropped into the complexing agent $\left(\mathrm{CaCl}_{2}\right.$ solution) again. The explants became at the center and covered with two layers of artificial endosperm. After $30 \mathrm{~min}$, calcium chloride was decanted and the beads were rinsed 3 times with autoclaved liquid multiplication medium for $15 \mathrm{~min}, 5 \mathrm{~min} / \mathrm{each}$, to wash away calcium chloride residues. The obtained beads were $7 \mathrm{~mm}-8 \mathrm{~mm}$ in diameter (Figure 2).

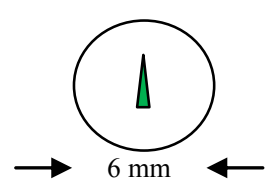

Figure 1. Single layered synseed.

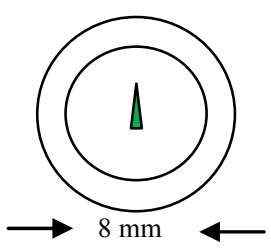

Figure 2. Double layered synseed with explant in the center of two layers of artificial endosperm. 


\subsubsection{Douple Layered Beads with Lateral Explant}

These type of beads were constructed by the method described by Kinoshita and Saito [36], where shoot tip explants and single layered beads (with or without $0.2 \%$ $(\mathrm{w} / \mathrm{v})$ activated charcoal) were completely immersed in sodium alginate medium with or without $0.2 \%(\mathrm{w} / \mathrm{v})$ activated charcoal. Then, they were together picked up and dropped into the complexing agent. The explant was included outside the inner layer but covered with the outer layer only. After $30 \mathrm{~min}$, calcium chloride solution was decanted and the beads were rinsed 3 times in sterilized multiplication medium to wash away $\mathrm{CaCl}_{2}$ residues. The obtained beads were about $9 \mathrm{~mm}$ in diameter (Figure 3).

\subsection{Conversion of Synseeds Influenced by Bead Type}

Shoot tip explants of Hindi cultivar encapsulated into ten types of synseeds were tested to determine the effect of bead type on synseed conversion. Single layered type synseed was with or without activated charcoal $(2 \mathrm{gm} / \mathrm{l})$. Two groups of double-layered synseeds were used: the first group with explant in the center of bead (Figure 2) and covered with two layers of artificial endosperm, and the second group with explants included outside the inner layer but covered only by the outer layer (Figure 3). In double-layered type, both two layers were with or without activated charcoal or one of them was supplemented with activated charcoal. All types of artificial seeds were conserved for one month as submerged beads in liquid multiplication medium at $4^{\circ} \mathrm{C}$ in refrigerator. The synseeds were converted on semisolid MS medium supplemented with $5 \mathrm{mg} / \mathrm{l} \mathrm{BAP}$ (conversion medium). A group of thirty synseeds of each type was cultured in six glass jar, five synseeds/each, and considered as a replicate for each type. After four weeks, percentage of synseed conversion, number of shoots per synseed, length of shoot and fresh weight per shoot cluster were determined.

\subsection{Effect of Explants Orientation on the Conversion of Synseeds}

In this experiment, one layer synseeds without charcoal were sued. A set of 90 encapsulated shoot tips was made and classified into three groups. Each group was cultured in six glass jars containing conversion medium. The first group was cultured where the orientation of shoot tip inside the beads was upward. The second group was

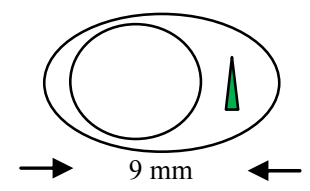

Figure 3. Double layered synseed with explants outside the inner layer but inside the outer layer. cultured where the shoot tip inside the bead was horizontally oriented. The third group was randomly cultured. After preservation for one month at $4{ }^{\circ} \mathrm{C}$ and incubation for more four weeks on conversion medium, percentage of converted synseeds, number of shoots per synseed, length of shoot and fresh weight per cluster were determined. After 4 days, peroxidase activity of explant inside the beads under conversion conditions was determined.

\subsection{Formation of Synseed Coat}

For synseed coating, the protocol proposed by Levy and Edwards-Levy [28] was used. The protocol consisted of the passage of shoot tip explants in artificial endosperm, which was prepared as previously described. The artificial endosperm solution was supplemented with $2.5 \%$ sodium alginate and after autoclaving, enriched with $2 \%$ PGA (Propylene glycole alginate, Kelcoloid $\mathrm{S}^{\circledR}$, Kelco International, $80 \%-85 \%$ esterification grade) and $5 \mathrm{ml} / 1$ of a patented antimicrobic PPM (Plant Preservative Mixture, Plant Cell Tech. Laboratories, USA). The solution was stirred for $24 \mathrm{~h}$ at room temperature to dissolve PGA. The complexing solution was made of the artificial endosperm solution with $\mathrm{CaCl}_{2}(75 \mathrm{mM})$. The coating solution was made of $50 \mathrm{ml}$ of artificial endosperm solution supplemented after autoclaving with $5 \mathrm{gm}$ egg albumin (10\% w/v final concentration). Encapsulation was made by immersing the explant with forceps in the first alginate-PGA solution and then dropping it in the stirred $\mathrm{CaCl}_{2}$ complexing solution. The encapsulated explants were kept stirred for 25 minutes and then transferred to a first rinse solution made of the simple artificial endosperm solution. After rinsing, the capsules were dropped in the coating solution, where they were left stirred for $5 \mathrm{~min}$, in order to allow penetration of the protein into the outer layer of the capsules. At this point, $2 \mathrm{ml}$ of $\mathrm{NaOH}(1 \mathrm{M})$ solution were added to the stirring solution with the capsules to start a transacylation reaction. The capsules were continuously stirred for a further $15 \mathrm{~min}$, then the solution was brought to $\mathrm{pH} 5.5$ with $2.5 \mathrm{ml} \mathrm{HCl}(1 \mathrm{M})$, and stirred for another $15 \mathrm{~min}$. After this, the capsules were finally rinsed in the artificial endosperm solution. In this experiment, all artificial endosperm solutions were made of conversion medium, and supplemented with $5 \mathrm{ml} / 1$ $\mathrm{PPM}^{\circledR}$. Forty coated synseeds were cultured in eight jars $(100 \mathrm{ml})$ contained semisolid multiplication medium 5 synseeds each After four weeks culture, percentage of synseed conversion, number of shoots per synseed, length of shoot and fresh weight per shoot cluster were determined.

\subsection{Determination of Peroxidase Activity}

Estimation of relative peroxidase activity was measured (O.D./gm fresh weight/h) and calculated according to 
Wakamatsu and Takahama [29]. For peroxidase analysis, $1 \mathrm{gm}$ of tissue was ground at $4^{\circ} \mathrm{C}$ in a mortar in $1 \mathrm{ml}$. extraction buffer consisting of $0.1 \mathrm{M}$ Tris-base $\mathrm{pH} 7.0$ and containing $0.002 \mathrm{M}$ cysteine. The homogenate was centrifuged at $14000 \mathrm{rpm}$ for $15 \mathrm{~min}$. The supernatant were collected for immediate peroxidase activity determination. The reaction mixture consisted of $5 \mathrm{mM}$ guaicol, $40 \mathrm{mM}$ potassium phosphate buffer, $\mathrm{pH} 7.2,0.1 \mathrm{mM}$ EDTA, $0.3 \mathrm{mM} \mathrm{H}_{2} \mathrm{O}_{2}$ and enzyme preparation $(50 \mu \mathrm{l}$ of each soluble peroxidase) in a final volume of $5 \mathrm{ml}$. The reaction was measured by the absorbance at $470 \mathrm{~nm}$ at the room temperature.

\section{Results and Discussion}

Sucker shoot tips were used for in vitro multiplication of banana on MS medium supplemented with $5 \mathrm{mg} / \mathrm{l}$ BAP. In order to save large number of plant materials to produce synseeds in large scale, six subcultures of in vitro obtained shoot tips were established. Banana shoot tips extracted from in vitro grown plant materials were big enough to facilitate the encapsulation procedure; consequently, great number of synseed could be formed in one hour (180 synseeds). The obtained capsules of synseeds were spherical and hard enough to be handled easily. Two types of synseeds, single layered and double layered synseeds were made. Single layered synseed was $5 \mathrm{~mm}$ $6 \mathrm{~mm}$ in diameter (Figure 1), however double layered one was $8 \mathrm{~mm}-9 \mathrm{~mm}$ in diameter (Figures 2 and $\mathbf{3}$ ).

Preliminary experiments showed that solution of $4 \%$ sodium alginate with $75 \mathrm{mM} \mathrm{CaCl} 2$ produced firm and clear uniform capsules within ion exchange duration in $30 \mathrm{~min}$. On the other side, higher concentrations of sodium alginate $(5 \%)$ were not suitable for synseed formation because the resulted beads were too hard to cause considerable delay in conversion and reduce the conversion frequency. Beads obtained from lower concentrations of sodium alginate $(3 \%)$ were too fragile to be handled. Ghosh and Sen [30] found that the use of high or low level of sodium alginate reduced the conversion frequency.

Conversion of banana synseeds influenced by bead type was studied by constructing several types of single and double layered synseeds with or without activated charcoal (Figures 4 and 5). They were cultured on conversion medium in order to determine the best synseed type. Induction of synseed conversion from encapsulated shoot tip explants was commenced after 3 days when explants were encapsulated in single layered synseeds with $0.2 \%$ activated charcoal. In case of single layered synseeds without charcoal and double layered synseeds (with explant covered with the outer layer and the inner layer with charcoal), the conversion of synseeds commenced in 4 days. In double layered synseeds with the explant in the outer layer and both layers either with or without charcoal, or only the outer layer with charcoal, the conversion of synseeds commenced in 5 days. On the other side, conversion of all types of double layered synseeds with explant in the center (covered with 2 endosperm layers) was delayed for 5 more days, where conversion commenced in 10 days.

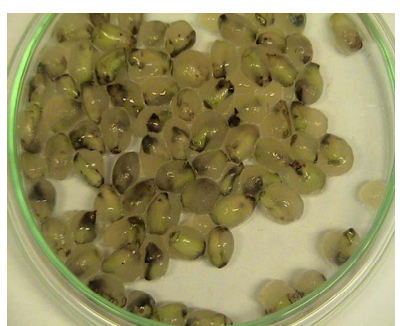

(a)

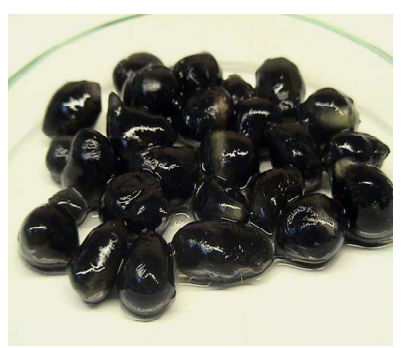

(d)

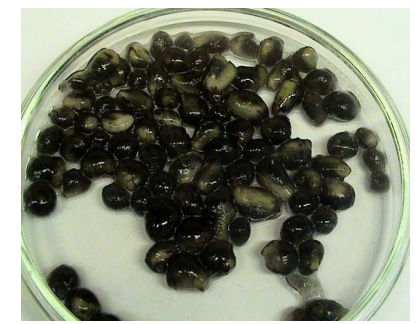

(b)

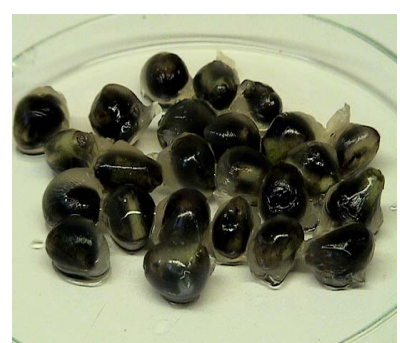

(e)

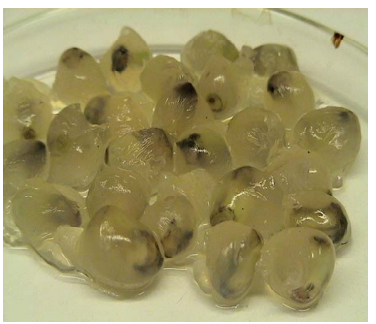

(c)

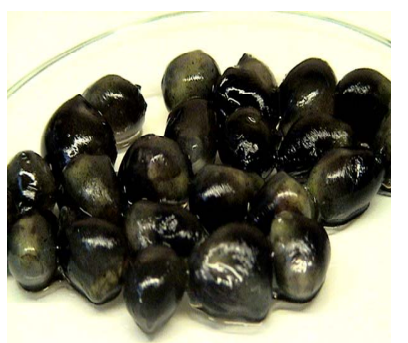

(f)

Figure 4. Different types of banana (Hnidi cultivar) synseeds with different types of artificial endosperm. (a) Single layer endosperm. (b) Single layer endosperm with charcoal. (c)-(f) Double layer synseed, explants were in the centers of the beads: (c) double layer endosperm, (d) double layer endosperm with charcoal, (e) double layer endosperm and the inner layer with charcoal, and (f) double layer endosperm and the outer layer with charcoal. 


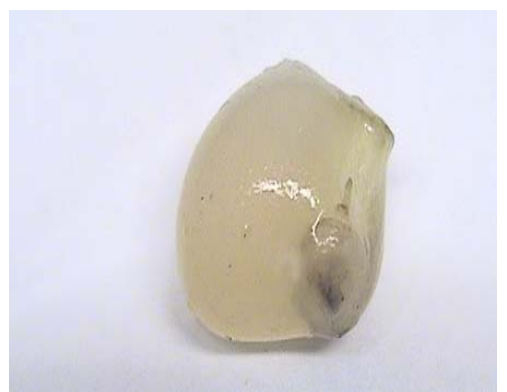

(a)

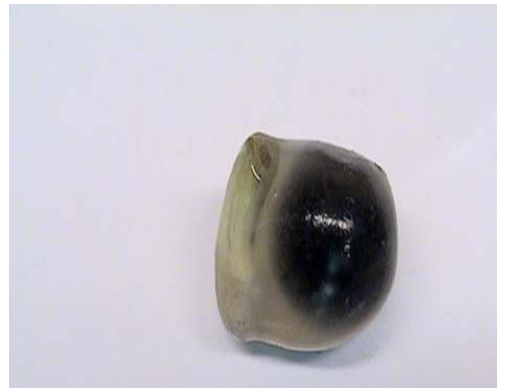

(c)

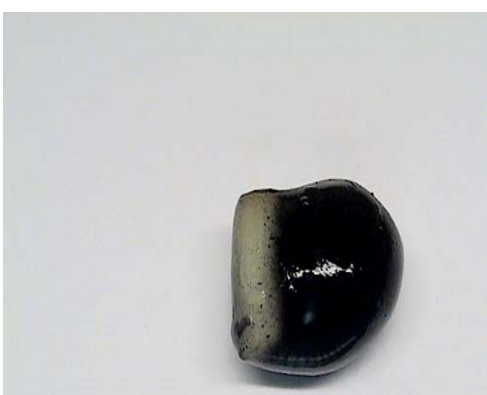

(b)

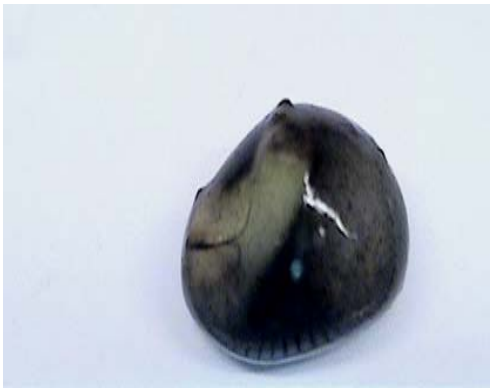

(d)

Figure 5. Double layer synseeds of banana (Hnidi cultivar) but the explant was coated with only one layer of endosperm, (a) without charcoal in both layers, (b) with charcoal in both layers, (c) with charcoal in the inner layer, and (d) with charcoal in the outer layer.

The results obtained after four weeks incubation on conversion medium indicated that the conversion of synseeds was affected by the number of endosperm layers, activated charcoal and the explant position inside the gel matrix. Single layered synseed with activated charcoal was the best construction in stimulating conversion of synseeds (Figure 6), where it expressed the highest percentage of synseed conversion, number of shoots, length of shoot and fresh weight/shoot cluster (Tables 1 and 2). Double layered synseeds with the explant in outer endosperm layer were better than those with the explant in the center and coated with two endosperm layers. The most effective type of double layered synseeds were those with explant in the outer layer and charcoal in both two layers (Table 2), where they expressed high percentage of synseed conversion, high shoot number, high shoot length and high fresh weight/shoot cluster.

Encapsulation of banana shoot tips in single layer was better than encapsulation in double layered synseeds. However, double layered synseeds with the explant in the outer endosperm layer with charcoal in both two layers were the most effective type where it expressed high conversion frequency especially under ex vitro condition (data not shown). This may be due to the ease of penetration and emergence of new shoots from one layer than from two layers. Micheli et al. [31] reported that sprouting of double layered synseeds was lower than that of single layered ones. Maruyama et al. [23] found that double layered synseeds were much better than single layered synseeds of Cidrela odorata L., Guazuma crinita Mart., and Jacaranda mimosaefolia D. Don.

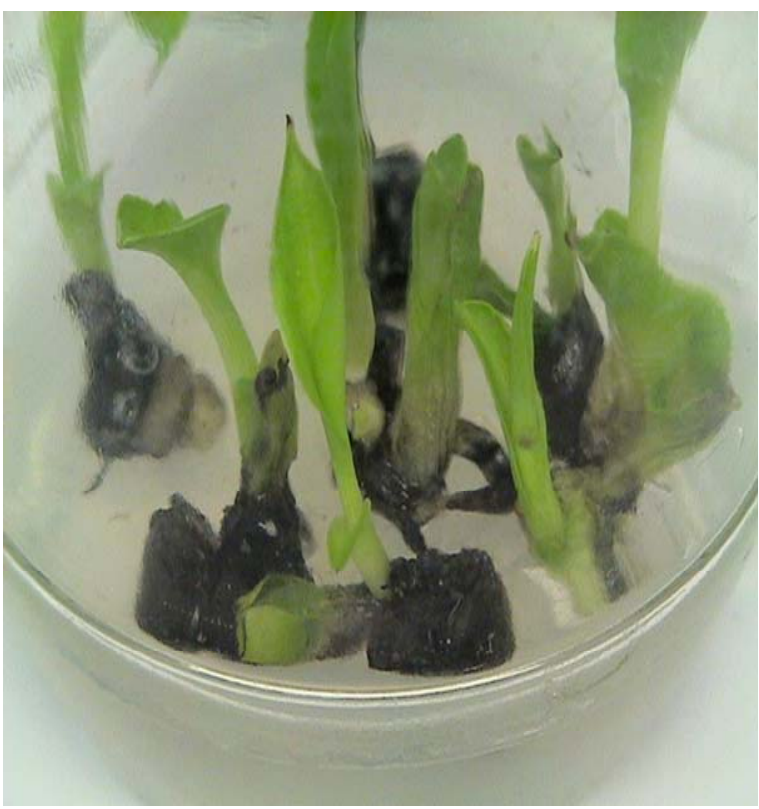

Figure 6. Conversion of banana single layer synseeds with charcoal. Synseeds were cultured on conversion medium for four weeks. 
Table 1. Conversion of encapsulated shoot-tip explants on conversion medium under the influence of explant position, activated charcoal, and number of endosperm layers. In case of double layered synseeds, the explant was encapsulated within two endosperm layers. Values are mean \pm SD.

\begin{tabular}{lcccc}
\hline \multicolumn{1}{c}{ Structure of synseed } & $\begin{array}{c}\text { Percentage of synseed } \\
\text { conversion (\%) }\end{array}$ & $\begin{array}{c}\text { No. of shoots/ } \\
\text { synseed }\end{array}$ & $\begin{array}{c}\text { Length of shoot } \\
\text { (cm) }\end{array}$ & $\begin{array}{c}\text { F.wt. } \\
\text { (gm) }\end{array}$ \\
\hline Single layer & 82.57 & $1.00 \pm 0.00$ & $3.85 \pm 0.17$ & $0.68 \pm 0.09$ \\
Single layer with charcoal & 98.34 & $2.17 \pm 0.38$ & $5.33 \pm 0.76$ & $0.87 \pm 0.04$ \\
Double layers & 75.00 & $1.50 \pm 0.54$ & $2.27 \pm 0.25$ & $0.38 \pm 0.04$ \\
Double layers with charcoal & 52.17 & $1.50 \pm 0.54$ & $3.07 \pm 0.11$ & $0.28 \pm 0.08$ \\
Double layers and the inner one with chacoal & 31.81 & $1.0 \pm 0.00$ & $0.98 \pm 0.08$ & $0.12 \pm 0.05$ \\
Double layers and the outer one with charcoal & 32.00 & $1.00 \pm 0.00$ & $2.43 \pm 0.40$ & $0.11 \pm 0.01$ \\
\hline
\end{tabular}

Table 2. Conversion of encapsulated shoot-tips on conversion medium under the influence of position of explants, activated charcoal, and number of endosperm layers. In case of double layered synseeds, the explant was encapsulated within one endosperm layer. Values are mean \pm SD.

\begin{tabular}{|c|c|c|c|c|}
\hline Structure of synseed & $\begin{array}{c}\text { Percentage of synseed } \\
\text { conversion }(\%)\end{array}$ & $\begin{array}{l}\text { No. of shoots/ } \\
\text { synseed }\end{array}$ & $\begin{array}{l}\text { Length of shoot } \\
(\mathrm{cm})\end{array}$ & $\begin{array}{l}\text { F.wt. } \\
\text { (gm) }\end{array}$ \\
\hline Single layer & 82.57 & $1.00 \pm 0.00$ & $3.85 \pm 0.17$ & $0.68 \pm 0.09$ \\
\hline Single layer with charcoal & 98.34 & $2.17 \pm 0.38$ & $5.33 \pm 0.76$ & $0.87 \pm 0.04$ \\
\hline Double layers & 79.16 & $1.00 \pm 0.00$ & $3.08 \pm 0.15$ & $0.36 \pm 0.05$ \\
\hline Double layers with charcoal & 90.00 & $1.50 \pm 0.54$ & $3.43 \pm 0.40$ & $0.66 \pm 0.05$ \\
\hline Double layers and the inner one with chacoal & 80.00 & $1.33 \pm 0.48$ & $2.75 \pm 0.20$ & $0.26 \pm 0.04$ \\
\hline Double layers and the outer one with chacoal & 72.00 & $1.00 \pm 0.00$ & $2.95 \pm 0.19$ & $0.25 \pm 0.04$ \\
\hline
\end{tabular}

The data in this work indicated that addition of activated charcoal into the gel matrix was beneficial because it improved the conversion as well as the number of shoots/synseed and shoot fresh weight. Vigorous growth was detected when the synthetic endosperm contained activated charcoal, where the shoot length, number of roots and root length were higher than those of synseeds without activated charcoal in synthetic endosperm. The beneficial effect of activated charcoal on synseeds conversion is attributed to their ability to absorb undesirable exudates, such as 5-hydroxymethylfurfural (a toxic breakdown product of sucrose formed during autoclaving) and other harmful phenolic oxidation products [23]. Also, it has been suggested that charcoal breaks up the alginate bead and thus increases respiration of somatic embryos, as well as it retains nutrients within the hydrogel capsule and slowly releases them to the growing embryo [32]. Furthermore, the addition of activated charcoal to the alginate beads matrix significantly enhanced root development and germination of encapsulated somatic embryos [33]. Our results showed that single layered synseed with $0.2 \%$ activated charcoal was the most effective synseed type for banana plants.

The previous reports indicated that conversion of synseeds depends on several factors where the conversion took place under in vitro or ex vitro conditions $[6,13,20]$. In this work, conversion of synseeds on a sterilized mixture of peat and sand $(2: 1 \mathrm{v} / \mathrm{v})$, and incubated under tissue culture conditions indicated that douple layered beads (especially where the explants covered only with one layer of artificial endosperm) were better than one layered beads (data not shown) but still it needs further studies to improve the ex vitro conversion of banana synseeds.

The effect of orientation of encapsulated shoot tip explants of Hindi cultivar on synseed conversion was shown in Table 3. Shoot tip explants were encapsulated in gel matrix without charcoal to determine the orientation of explant on medium. The highest conversion frequency was obtained when the direction of the shoot tip explant inside synthetic endosperm was upward and its base was in contact with the medium surface. It was accompanied by high increase in peroxidase activity. The lowest conversion frequency was obtained when the 
shoot tip explants inside the synthetic endosperm was in horizontal position on conversion medium. The polarity of auxin transport is basipetal, i.e., auxin moves from apex to base region. When the encapsulated explants were placed in upward position on conversion medium, auxins transported from the apex of explant to its base, and in the same time the explant absorbed the cytokinin from the conversion medium. Consequently, right cytokinin/auxin ratio was established to induce cell division and led to conversion of encapsulated explants. So, when the encapsulated explant was in horizontal position, the portion of auxins that was flowed towards lower side of the explant under the influence of gravity and diffused to the medium [34]. In this case, the remained auxin in the explant became low than that needed to establish the right auxin/cytokinin ratio resulting in repressing the synseed conversion. In random culture, some of the encapsulated explants may be in upward direction and others may be in horizontal direction or in between, thus their conversion frequency was better than horizontal culture but lower than upward culture.

Single layered synseeds were subjected for seed coating procedure. Synthesis of seed coats did not change the size of beads or their appearance as well as their consistence. Consequently, it was difficult to distinguish between synseeds with or without seed coats (Figure 7). Charcoal was not included in single layered synseed when seed coats were synthesized to cover the artificial endosperm. Culture of synseeds with or without coat on conversion medium for four weeks indicated that there was a big difference between the data of uncoated and coated synseeds. Coated synseeds expressed higher conversion frequency, shoot length and fresh weight of shoot cluster than those obtained by uncoated ones (Table 4). It's worthy to mention that synthesis of seed coat stimulate not only shoot but also root formation (Figure 8) and the resulted plantlets transferred to ex vitro conditions with limited success.

Table 3. Effect of orientation of encapsulated explants cultured on conversion for four weeks on conversion and peroxidase activity of encapsulated explants. Values are mean \pm SD.

\begin{tabular}{ccccccc}
\hline Orientation & $\begin{array}{c}\text { Conversion frequency } \\
(\mathbf{\%})\end{array}$ & $\begin{array}{c}\text { No. of } \\
\text { shoots/synseed }\end{array}$ & $\begin{array}{c}\text { Length of shoot } \\
\text { (cm) }\end{array}$ & $\begin{array}{c}\text { F.wt. } \\
\text { (gm) }\end{array}$ & $\begin{array}{c}\text { POX activity } \\
\text { (\%) }\end{array}$ \\
\hline Random & 51.42 & $1.82 \pm 0.79$ & $3.91 \pm 0.39$ & $0.48 \pm 0.07$ & 100 \\
Upward & 67.65 & $3.50 \pm 0.58$ & $4.26 \pm 0.25$ & $0.65 \pm 0.04$ & $348.12 \pm 11.69$ \\
Horizontal & 42.85 & $1.25 \pm 0.5$ & $3.73 \pm 0.05$ & $0.46 \pm 0.08$ & $92.11 \pm 6.91$ \\
\hline
\end{tabular}

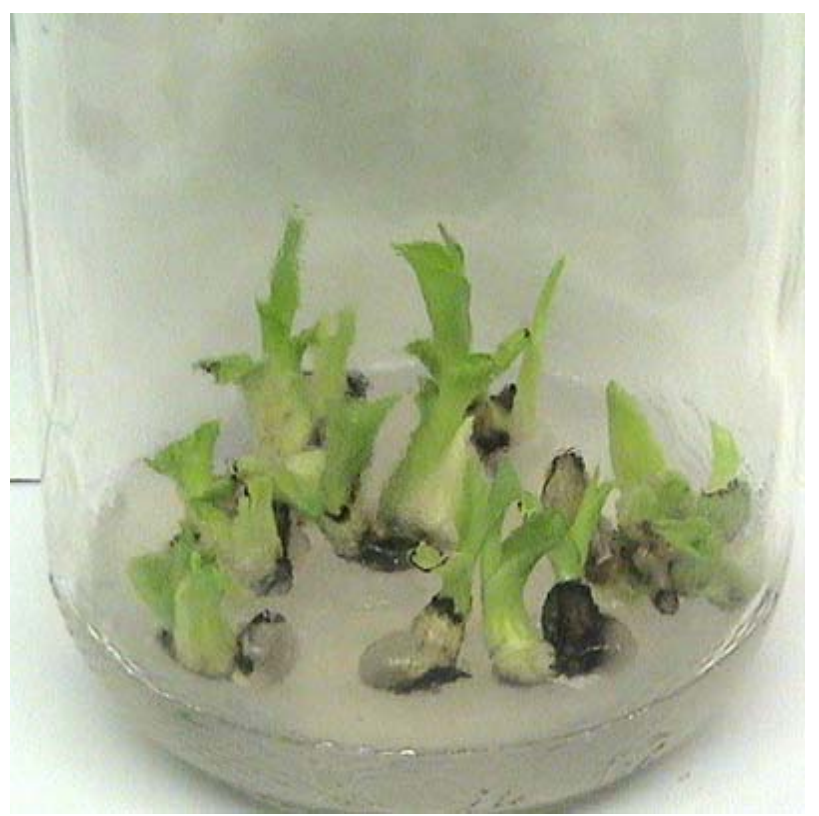

Figure 7. Conversion of coated single layer synseeds of Hindi cultivar cultured for four weeks on conversion medium.

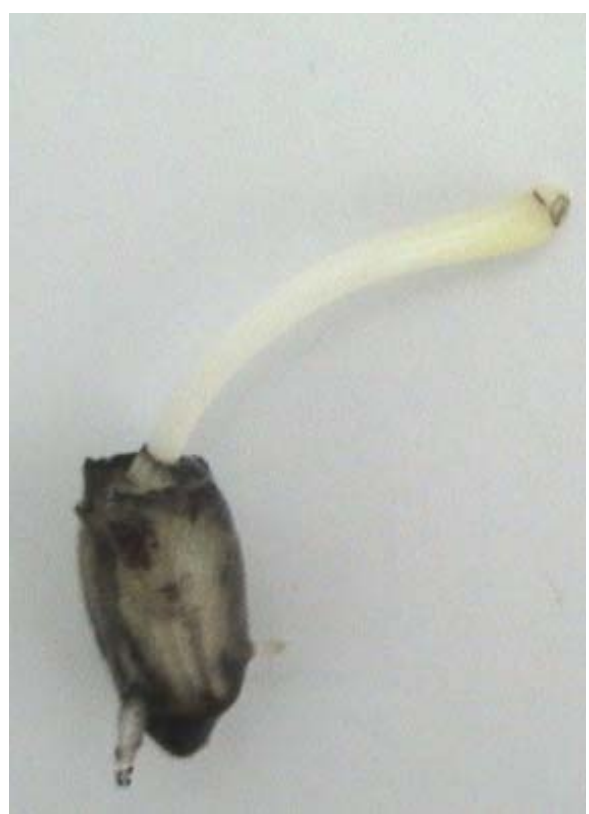

Figure 8. Conversion of coated synseeds in Petri dish at $25^{\circ} \mathrm{C}$. 
Table 4. Effect of coating on the conversion of synseeds of banana. Synseeds were cultured for four weeks on conversion medium. Values are mean \pm SD.

\begin{tabular}{rcccc}
\hline Type of synseed & Conversion frequency (\%) & Shoot no./synseed & Shoot length (cm) & F.wt. (gm) \\
\hline Synseed without coat & 81.81 & $1.25 \pm 0.50$ & $3.00 \pm 0.39$ \\
Synseed with seed coat & 96.77 & $4.00 \pm 0.89$ & $0.37 \pm 0.05$ & $2.03 \pm 0.11$ \\
\hline
\end{tabular}

Hydrophobic thin coat over a gel surface plays an important role of preventing the rupture and rapid desiccation of synseeds [35]. It also expected that coat acts as a shield against microbial and chemical contamination of synseeds. Consequently, coating of the encapsulated banana shoot tips was established to determine the advantages of coating on banana synseed conversion. Our data indicated that, the conversion frequency, number of shoots/ synseed and shoot length increased when the gel matrix was coated. This indicated that the used method for formation of synseed coat did not affect negatively on the studied parameters. Redenbaugh et al. [36] reported that coating did not reduce the efficiency of shoot emergence from alfalfa somatic embryos.

This work described efficient procedure to produce artificial synseed in large scale in banana where six subcultures of in vitro obtained shoot tips were established. Encapsulation of shoot tips provided reliable method to produce synseeds. Single layered synseeds with charcoal expressed the highest conversion frequency. Creation of seed coat around the artificial endosperm was recommended for synseed conversion. In addition, to improve synseed conversion on conversion substratum shoot tips should be oriented to be upward.

\section{REFERENCES}

[1] S. Cronauer and A. D. Krikorian, "Multiplication of Musa from Excised Stem Tip," Annals of Botany, Vol. 53, 1984, pp. 321-328.

[2] O. Arias, "Commercial Micropropagation of Banana," In: San Jose and Costa Rica, Eds., Biotechnology Applications for Banana and Plantain Improvement, The International Network for the Improvement of Banana and Plantain (INIBAP), 1992, pp. 139-142.

[3] L. Sagi, D. M. George, S. Remy and R. Swennen, "Recent Developments in Biotechnological Reseach on Bananas (Musa spp.)," Biotechnology \& Genetic Engineering Reviews, Vol. 15, 1998, pp. 313-317.

[4] N. Jafari, R. Y. Othman and N. Khalid, "Effect of Benzylaminopurine (BAP) Pulsing on in vitro Shoot Multiplication of Musa acuminata (Banana) cv. Berangan," African Journal of Biotechnology, Vol. 10, No. 13, 2011, pp. 2446-2450.

[5] S. C. Hwang, C. L. Chen, J. C. Lin and H. L. lin, "Cultivation of Banana Using Plantlets from Meristem Cul- ture," HortScience, Vol. 19, 1984, pp. 231-233.

[6] F. Jaseela, V. R. Sumitha and G. M. Mair, "Somatic Embryogenesis and Plantlet Regeneration in an Agronomically Important Wild Rice Species Oryza nivara," Asian Journal of Biotechnology, Vol. 1, 2009, pp. 74-78.

[7] S. Khan, B. Saeed and N. Kauser, "Establishment of Genetic Fidelity of in vitro Raised Banana Plnatlets," Pakistan Journal of Botany, Vol. 43, No. 1, 2011, pp. 233242.

[8] M. J. M. Smulders and G. J. de Klerk, "Epigenetics in Plant Tissue Culture," Plant Growth Regulation, Vol. 63, No. 2, 2011, pp. 137-146. doi:10.1007/s10725-010-9531-4

[9] R. L. M. Pierik, "Commercial Micropropagation in Western Europe and Israel," In: P. C. Debergh and R. H. Zimmerman, Eds., Micropropagation: Technology and Application, Kluwer Academic Publisher, Dordrecht, 1991, pp. 155-166.

[10] P. C. Debergh and P. E. Read, "Micropropagation," In: P. C. Debergh and R. H. Zimmerman, Eds., Micropropagation: Technology and Application, Kluwer Academic Publisher, Dordrecht, 1991, pp. 1-14.

[11] S. L. Kitto, "Commercial Micropropagation," HortScience, Vol. 32, 1997, pp. 1012-1014.

[12] T. Ivaylo and J. Hausman, "In vitro Regeneration from Alginate-Encapsulated Microcuttings of Quercus sp.," Scientia Horticulturae, Vol. 103, 2005, pp. 503-507. doi:10.1016/j.scienta.2004.06.013

[13] A. Ray and S. Bhattacharya, "Storage and Plant Regeneration from Encapsulated Shoot Tips of Rauvolfia serpentina-An Effective Way of Conservation and Mass Propagation," South African Journal of Botany, Vol. 74, No. 4, 2008, pp. 776-779. doi:10.1016/j.sajb.2008.06.002

[14] T. R. Ganapathi, P. Suprasanna, V. A. Bapat and P. S. Rao, "Propagation of Banana through Encapsulated Shoot Tips," Plant Cell Reports, Vol. 1, 1992, pp. 571-575.

[15] N. Neives, J. C. Lorenzo, M. A. Blanco, J. Gonzalez, H. Peralta, M. Hernandez, R. Santos, O. Conceptio, C. G. Borroto, E. Borrto, R. Tapia, M. E. Martinez, Z. Fundora and A. Gonzalez, "Artificial Endosperm of Cleapatra tangerine Zygotic Embryos: A Model for Somatic Embryo Encapsulation," Plant Cell, Tissue and Organ Culture, Vol. 54, No. 2, 1998, pp. 77-83. doi:10.1023/A:1006101714352

[16] T. R. Ganapathi, L. Sprinivas, P. Suprasanna and V. A. Bapat, "Regeneration of Plants from Alginate-Encapsulated Somatic Embryos of Banana cv. Rasthali (Musa spp. AAB Group)," In Vitro Cellular \& Developmental Biol- 
ogy Plant, Vol. 37, No. 2, 2001, pp. 178-181. doi:10.1007/s11627-001-0031-0

[17] E. Maruyama, K. Ishii, I. Kinoshita, K. Ohba and A. Saito, "Micropropagation of Bolaina Blanca (Guazuma crinita Mart.), a Fast-Growing Tree in the Amazon Region," Journal of Forestry Research, Vol. 1, 1996.

[18] S. Pattnaik and P. K. Chand, "Morphogenic Response of the Alginate-Encapsulated Axillary Buds from in vitro Shoot Cultures of Six Mulberries," Plant Cell, Tissue and Organ Culture, Vol. 60, No. 3, 2000, pp. 177-185. doi:10.1023/A:1006424626433

[19] R. Mahanraj, R. Ananthan and V. N. Bai, "Production and Storage of Synthetic Seeds in Coelogyne breviscapa Lindl.," Asian Journal of Biotechnology, Vol. 1, 2009, pp. 124-128.

[20] D. K. Samah, M. Barthakur and P. K. Borua, "Artificial Seed Production from Encapsulated PLBs Regenerated from Leaf Base of Vanda coerulea Griff. Ex. Lindl.-An Endangered Orchid," Current Science, Vol. 98, 2010, pp. 686-690.

[21] W. Pimada and S. Bunnag, "Cryopreservation of Dendrobium heterocarpum Lindl. via Encapsulation Method," ELBA Bioflux, Vol. 2, 2010, pp. 7-14.

[22] B. D. McKersie and S. R. Bowley, "Artificial Seeds of Alfalfa," In: K. Redenbaugh, Ed., Synseeds, 235 California, 1993.

[23] E. Maruyama, I. Kinoshita, K. Ishii, H. Shigenaga, K. Ohra and A. Saito, "Alginate-Encapsulated Technology for the Propagation of the Tropical Forest Trees: Cedrela odorata L., Guazuma crinita Mart., and Jacaranda mimosaefolia D. Don," Silvae Genetica, Vol. 46, 1997, pp. 17-23.

[24] V. A. Bapat, "Studies on Synthetic Seeds of Sandalwood (Santalum album L.) and Mulberry (Morus indica L.)," In: K. Redenbaugh, Ed., Synseeds: Application of Synthetic Seeds to Crop Improvement, CRC, Boca Raton, Fla., 1993, pp. 381-407.

[25] T. Gardi, E. Piccioni and A. Standardi, "Effect of Bead Nutrient Composition on Growth Ability of Stored vitroDerived Encapsulated Microcuttings of Different Woody Species," Journal of Microencapsulation, Vol. 1, 1998, pp. 13-25.

[26] E. Piccioni and A. Standardi, "Encapsulation of MicroPropagated Buds of Six Woody Species," Plant Cell, Tissue and Organ Culture, Vol. 42, No. 3, 1995, pp. 221-226.

\section{doi:10.1007/BF00029990}

[27] T. Murashige and F. Skoog, "A Revised Medium for Rapid Growth and Bioassay with Tobacco Tissue Cultures," Physiologia Plantarum, Vol. 15, 1962, pp. 473-497.

[28] M. C. Levy and F. Edwards-Levy, "Coating Alginate Beads with Cross-Linked Bipolymers: A Novel Method Based on a Transaclation Reaction," Journal of Microecapsulation, Vol. 13, 1996, pp. 169-183.

[29] K. Wakamatsu and U. Takahama, "Changes in Peroxidase Activity and Peroxidase Isoenzyme in Carrot Callus," Plant Physiology, Vol. 88, 1993, pp. 167-171. doi:10.1034/j.1399-3054.1993.880123.x

[30] B. Ghosh and S. Sen, "Plant Regeneration from Alginate Encapsulated Somatic Embryos of Asparagus cooperi Baker," Plant Cell Reports, Vol. 13, No. 7, 1994, pp. 381-385. doi:10.1007/BF00234142

[31] M. Micheli, A. Stadardi, P. Dell'Orco and M. Mencuccini, "Preliminary Studies on the Synthetic Seed and Encapsulation Technologies of vitro-Derived Olive Explants," In: C. Vitagliano, G. P. Martelli, Eds., Proceedings of the 4th IS on Olive Growing, Acta Horti, 586, ISIIS, 2002.

[32] G. V. S. Saiprasad, "Artificial Seeds and Their Applications," Resonance, 2001, pp. 39-47.

[33] M. M. Lulsdorf, T. E. Tautorus, S. I. Kikcio, T. D. Bethune and D. I. Dunstan, "Germination of Encapsulated Embryos of Interior Spruce (Picea glauca engelmannii Complex) and Black Spruce (Picea mariana MILL)," Plant Cell Reports, Vol. 12, No. 7-8, 1993, pp. 385-389. doi:10.1007/BF00234697

[34] F. W. Went, "Wuchstoff Und Wachstum," Recueil des Travaux Botaniques Néerlandais, Vol. 25, 1928, pp. 1116.

[35] J. A. A. Fujii, D. T. Slade, K. Redenbaugh and K. A. Walker, "Artificial Seeds for Plant Propagation," Biotechnology, Vol. 5, 1987, pp. 335-339.

[36] K. Redenbaugh, D. Slade, P. Viss and J. A. Fujii, "Encapsulation of Somatic Embryos in Synthetic Seed Coats," Horticultural Science, Vol. 22, 1987, pp. 803-809.

[37] I. Kinoshita and A. Saito, "Regeneration of Japanese White Birch Plants from Encapsulated Axillary Buds," In: M. Kuwahara and M. Shimada, Eds., Proceedings of the 5th International Conference on Biotechnology in the Pulp and Paper Industry, Kyoto-Japan, UniPublishers, Tokyo, 1992, pp. 493-496. 